\title{
Study of Transparency of Pigments to Near Infrared ${ }^{+}$
}

\author{
Marija Strojnik* and Manuel Bravo-Medina
}

Optical Research Center, Apdo. Postal 1-948, 37000 Leon, Gto., Mexico; beethoven@cio.mx

* Correspondence: mstrojnik@gmail.com; Tel.: +1480-294-7419

+ Presented at the 15th International Workshop on Advanced Infrared Technology and Applications (AITA 2019), Florence, Italy, 17-19 September 2019.

Published: 10 October 2019

\begin{abstract}
We describe the results of a systematic study of reflectance of pigments traditionally used in Mexican cultural treasures on different substrates. We observe that underline drawings may be most easily detected in NIR when the pigment transmissivity in NIR is high. We conclude that the under-drawings of best quality are detected with illuminators with low NIR wavelengths.
\end{abstract}

Keywords: pigments; transparency; NIR; substrate; cultural heritage; IR illuminators

\section{Introduction}

Mexico has a rich and diverse cultural heritage. Most of that heritage is very rather old; it has been neglected for a long time, it has been physically subject to various civil wars; and thus, it is poorly documented. We are interested in using near infrared radiation (NIR) to determine the historical value of unknown pieces of national heritage. We are attempting to classify local materials as to their transparency in NIR when used on canvas.

We appreciate that the coloring agents depend on availability of local dyes. This field has been amply studied in Italian art, with results presented in Figure 1. IR reflectography can help visualize hidden details under painting layers, which can provide valuable information for art historians researching a piece: Has it been reconstructed before? Do under-drawings indicate different objectives of the owners of the paintings during different times?

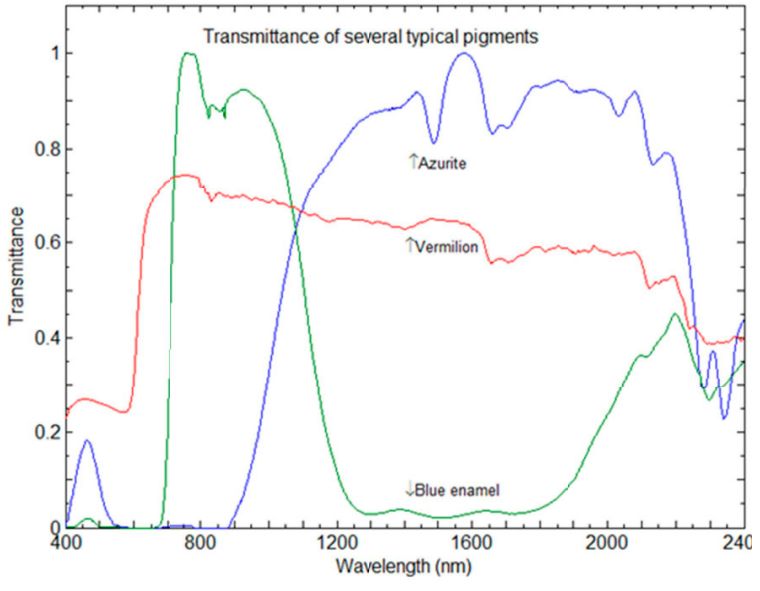

(a) $[0.40 \mu \mathrm{m}-2.40 \mu \mathrm{m}]$

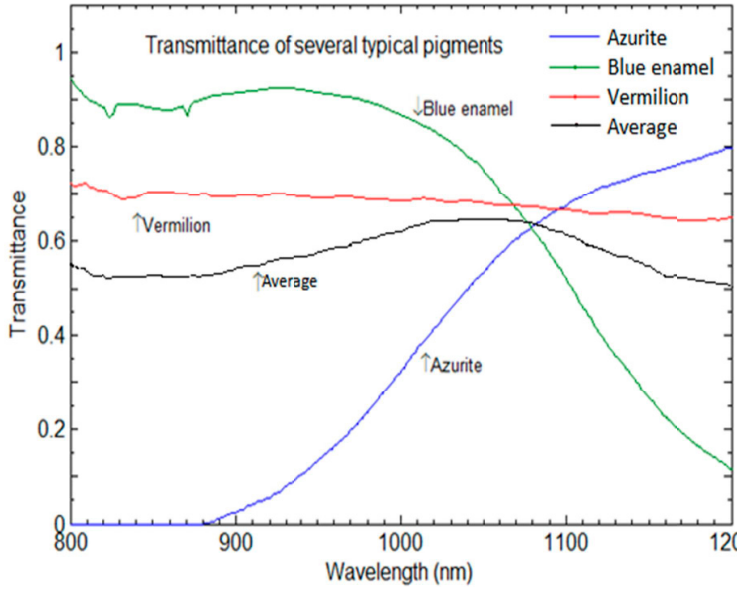

(b) $[0.80 \mu \mathrm{m}-1.20 \mu \mathrm{m}]$

Figure 1. Transmittance of several typical paints, used historically in Italy in spectral region $[0.80 \mu \mathrm{m}$ $-1.20 \mu \mathrm{m}$ ], modified after [1], with permission. All three transmittance curves have about the same value of 0.65 at wavelength $1.080 \mu \mathrm{m}$. The black transmittance curve [denoted average in part (b)] shows the average paint obtained by adding up all three contributions and dividing it by 3 . It has a broad peak at about $1.050 \mu \mathrm{m}$. 


\section{Materials and Methods}

Pigments tend to be based on the raw materials available in the geographical location of the author's workshop. NIR transparency depends not only on the pigment, but also on the substrate used to fix it to the canvas. Only a few pigments are well documented, so it is not always easy to choose the optimal NIR wavelength for the reflectogram.

The objective of this work is to determine the NIR transparency of several pigments typically employed in the Nueva España (Mexico) region. Additionally, we studied the reflection spectrum of these pigments to try to correlate them with their transparency.

We employed two pigment matrices: for color pigments (C) and for brown pigments (B), shown in Figure 2. On B, each column on each matrix features a different pigment and each row features a different substrate. On $\mathrm{C}$, each row is a different pigment and each column a different substrate. On some elements, the under-drawing even in the visible is noticeable. This is quite noticeable for the color matrix, elements 1.2 through 1.5, and the blue 4.4. The dark blue color pixel, denoted 4,3, appears visually quite opaque.

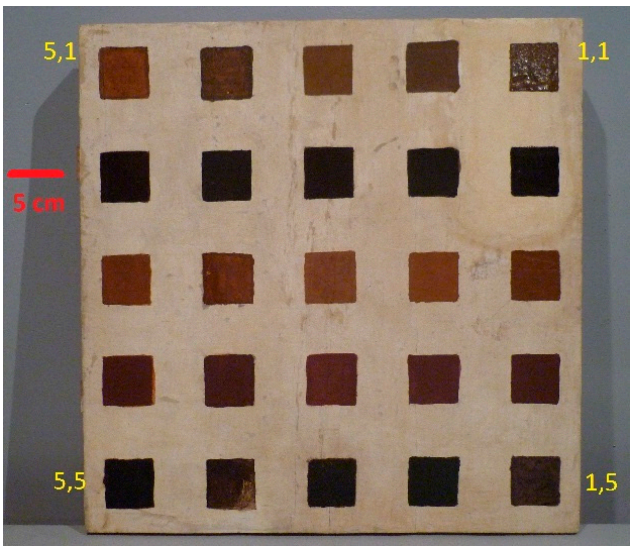

(a) Matrix with brown pigments (B)

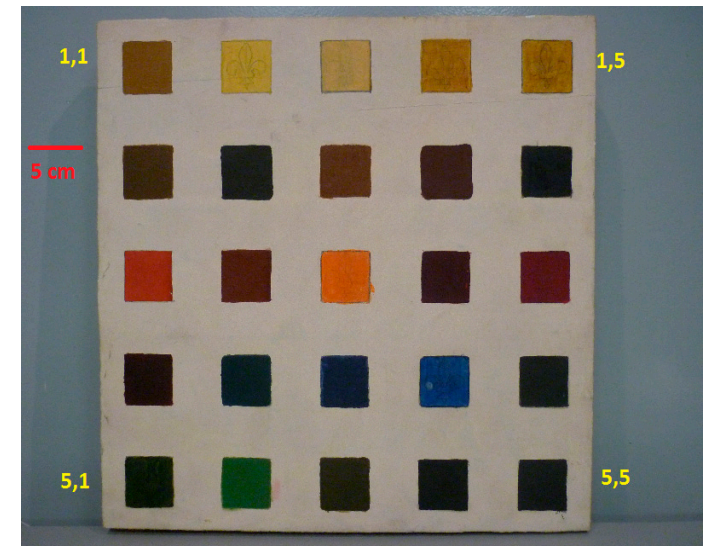

(b) Matrix with color pigments (C)

Figure 2. Pigment matrices: for color pigments (C) and for brown pigments (B). On B, each column on each matrix features a different pigment and each row features a different substrate. On $\mathrm{C}$, each row is a different pigment and each column a different substrate. On some elements, the underdrawing even in the visible is noticeable. This is quite apparent for the color matrix, elements 1.2 through 1.5, and the blue 4.4.

\section{Experimental}

Figure 3 presents the image of color matrix as captured by Xenics-XS camera on the left and its spectral responsivity on the right.

We measure the reflectance spectra of each matrix element using a fiber optic spectrometer fitted to an integrated sphere with a tungsten halogen lamp. The spectra are compared to the reflectance spectra of a $99 \%$ reflectivity reflectance standard. We also measured the reflectance spectrum of the canvas in order to compare it with the matrix elements.

We captured NIR reflectograms of each matrix element using an InGaAs camera. To achieve multispectral imaging, we recorded several for each matrix element using different illumination sources. The narrow spectral width sources include four different 10-LED NIR lamps (centered on 970, 1050, 1070 and $1200 \mathrm{~nm}$ ) with a FWHM of $100 \mathrm{~nm}$, and three different high power LED lamps (centered on 1300, 1450 and $1550 \mathrm{~nm}$ ) with a FWHM of $100 \mathrm{~nm}$. We also captured reflectograms with an incandescent light source illuminator (blackbody-like radiation). 


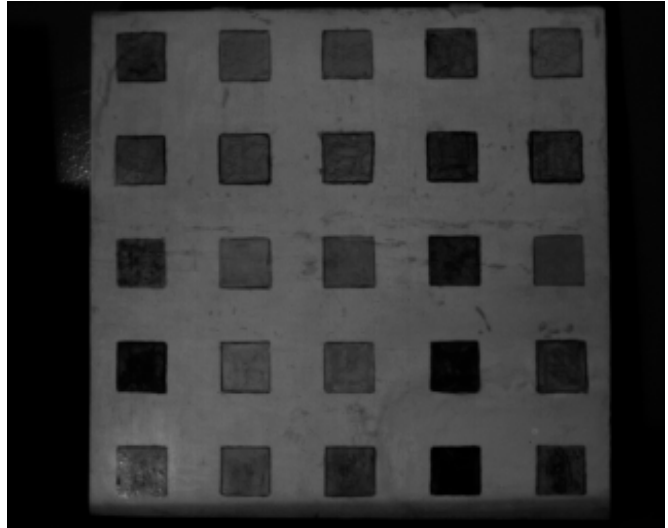

(a) Color matrix captured by Xenics-XS camera.

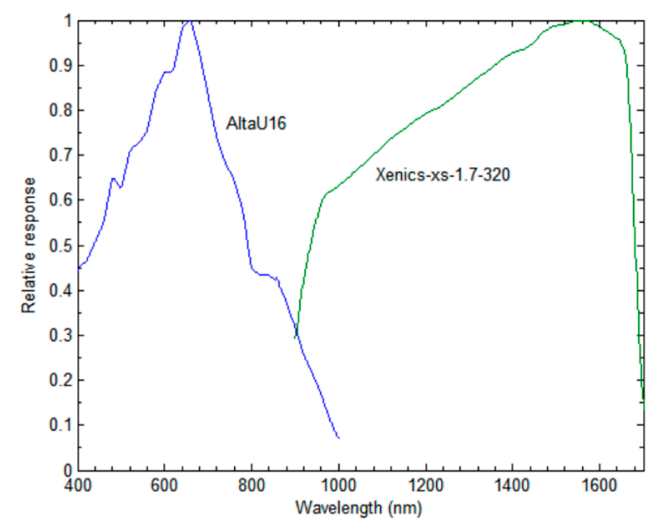

(b) Xenics-XS and AltaU16 responsivity curves.

Figure 3. (a) Color matrix as seen by Xenics-XS camera; (b) Responsivities of two cameras from 800 $\mathrm{nm}$ to $1700 \mathrm{~nm}$, with peaks normalized: the LLL intensified AltaU16 and the Xenics-XS.

\section{Results}

The measured spectral reflectance of canvas and transparent pixels in row 1 of color matrix are shown in Figure 4a. With the exception of the first pixel that is indeed opaque in the visible, all the transparent pixels seem to have the same spectral form, except that the curves appear to be shifted to lower transmittance values with the increasing pixel number (see Figure $2 b$ ). Figure $4 b$ shows a photo of a representative ten-LED source.

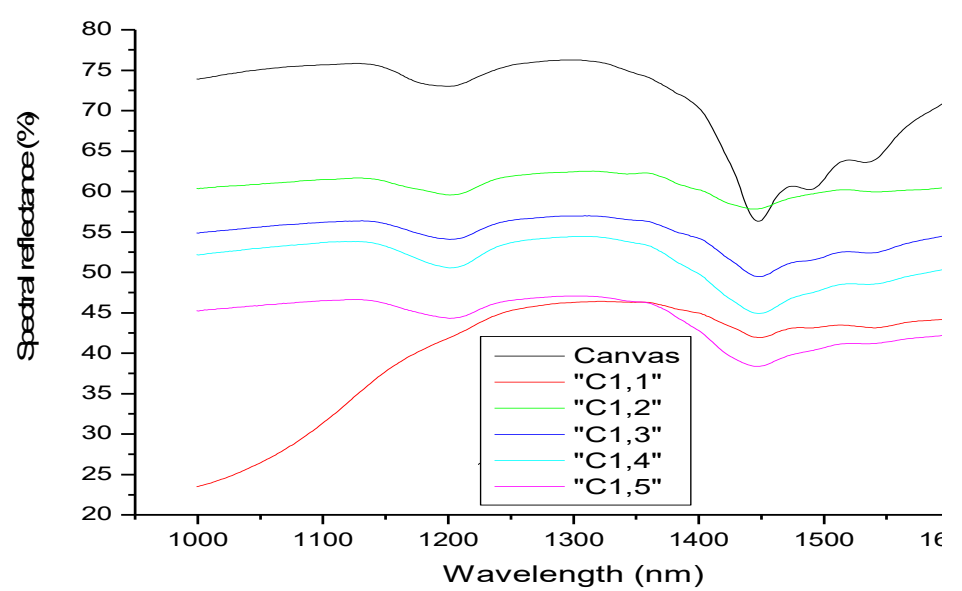

(a) Measured reflectance of canvas and transparent color pixels.

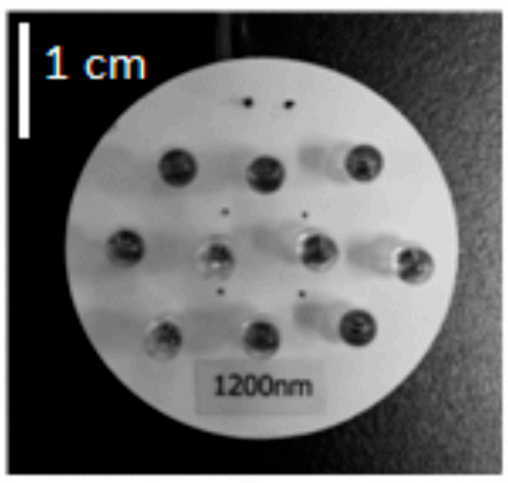

(b) Ten-LED source at $1200 \mathrm{~nm}$.

Figure 4. Measured reflectance signature depends on the spectral content of the source of illumination and the reflecting surface. (a) Measured reflectance of canvas and transparent color pixels; (b) TenLED source at $1200 \mathrm{~nm}$ [2].

We compared the transparency of each element for each lamp in Figure 5. We present different detected reflectances from the colored pixel 4,3, indicated dark blue when illuminated with the IR radiation. The central wavelength of the illuminating lamp is indicated below the sample. 


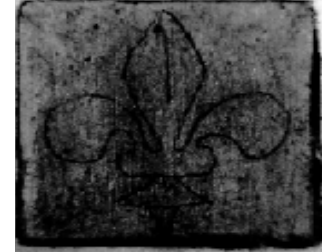

$1050 \mathrm{~nm}$

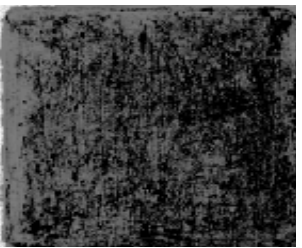

$1300 \mathrm{~nm}$

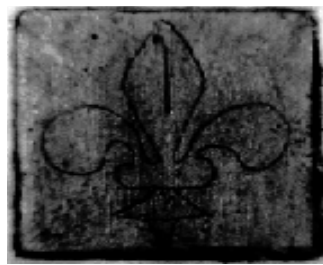

$970 \mathrm{~nm}$

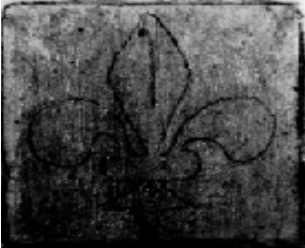

$1070 \mathrm{~nm}$

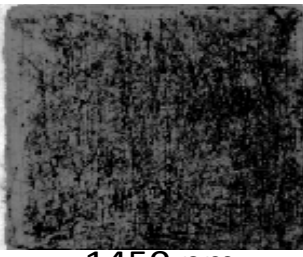

$1450 \mathrm{~nm}$

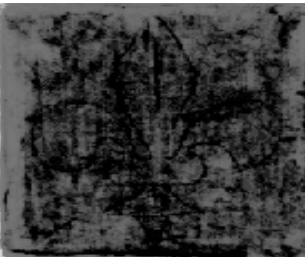

Incandescent

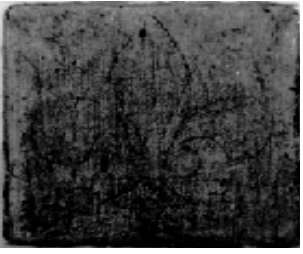

$1200 \mathrm{~nm}$

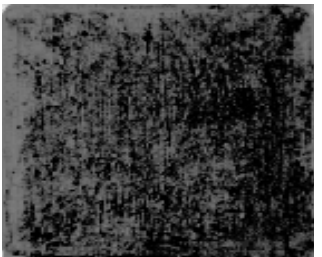

$1550 \mathrm{~nm}$

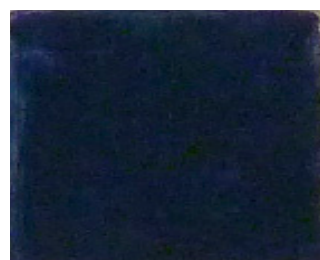

Color 4,3

Figure 5. Multispectral imaging of the Color pixel 4,3 (lower right corner) indicates dark blue color observed in the visible. Different degrees of transparency are indicated when this pixel is illuminated with the IR radiation. The central wavelength in $\mathrm{nm}$ of the illuminating lamp is indicated below the sample, ranging from 1050 to $970 \mathrm{~nm}$. The best quality of the underline image is obtained with illumination at $970 \mathrm{~nm}$. The image quality decreases with increasing wavelength.

\section{Conclusions}

Substrate significantly alters the pigment spectrum, obscuring some spectral regions. The pigment is not transparent in the spectral regions where the pigment reflectance is much lower than that of the canvas.

Reflectance spectra of low transparency pigments tend to be flatter in form, while the spectra of high transparency paints had a shape similar to that of the canvas spectrum. The analyzed pigments exhibit decreased transparency with increasing wavelengths.

Author Contributions: The second author contributed image processing, the first author conceptualization, analysis and writing.

Funding: The funding reported in this research was provided by the CONACYT.

Acknowledgments: Authors acknowledge helpful discussions with Gonzalo Paez.

Conflicts of Interest: The authors declare no conflict of interest.

\section{References}

1. Gargano, M.; Ludwig, N.; Milazzo, M., Petrucci, F. Recent developments of instruments for infrared reflectographic analysis of paintings. Adv. Infrared Technol. Appl. 2007, 407-415.

2. Strojnik, M.; Ortega, A. Near IR diodes as illumination sources to remotely detect under-drawings on century-old paintings. In Proceedings of the 22nd Congress of the International Commission for Optics: Light for the Development of the World, Puebla, Mexico, 25 October 2011; doi:10.1117/12.902160. 